OPEN ACCESS

Edited by:

Evangelos P. Misiakos, University of Athens School of

Medicine, Greece

Reviewed by:

Scott R. Kelley,

Mayo Clinic, USA

Juan Manuel Suárez-Grau,

General Hospital of Riotinto, Spain

${ }^{*}$ Correspondence:

Neil J. Smart

drneilsmart@hotmail.com

Specialty section:

This article was submitted to Visceral Surgery,

a section of the journal

Frontiers in Surgery

Received: 27 July 2015

Accepted: 28 January 2016

Published: 16 February 2016

Citation:

Alam NN, Narang SK, Köckerling F,

Daniels IR and Smart NJ (2016) Biologic Mesh Reconstruction of the

Pelvic Floor after Extralevator

Abdominoperineal Excision: A

Systematic Review.

Front. Surg. 3:9.

doi: 10.3389/fsurg.2016.00009

\section{Biologic Mesh Reconstruction of the Pelvic Floor after Extralevator Abdominoperineal Excision: A Systematic Review}

\author{
Nasra N. Alam ${ }^{1}$, Sunil K. Narang ${ }^{1}$, Ferdinand Köckerling ${ }^{2}$, lan R. Daniels ${ }^{1}$ and \\ Neil J. Smart ${ }^{1 *}$ \\ ${ }^{1}$ Exeter Surgical Health Services Research Unit (HeSRU), Royal Devon and Exeter Hospital, Exeter, Devon, UK, ${ }^{2}$ Department \\ of Surgery, Center for Minimally Invasive Surgery, Academic Teaching Hospital of Charité Medical School, Vivantes Hospital, \\ Berlin, Germany
}

Introduction: The aim of this review is to provide an overview of the evidence for the use of biologic mesh in the reconstruction of the pelvic floor after extralevator abdominoperineal excision of the rectum (ELAPE).

Methods: A systematic search of PubMed was conducted using the search terms: "ELAPE," "extralevator abdominoperineal excision of rectum," or "extralevator abdominoperineal resection." The search yielded 17 studies.

Results: Biologic mesh was used in perineal reconstruction in 463 cases. There were 41 perineal hernias reported but rates were not consistently reported in all studies. The most common complications were perineal wound infection $(n=93)$, perineal sinus and fistulae $(n=26)$, and perineal haematoma or seroma $(n=11)$. There were very few comparative studies, with only one randomized control trial $(\mathrm{RCT})$ identified that compared patients undergoing ELAPE with perineal reconstruction using a biological mesh, with patients undergoing a conventional abdominoperineal excision of the rectum with no mesh. There was no significant difference in perineal hernia rates or perineal wound infections between the groups. Other comparative studies comparing the use of biologic mesh with techniques, such as the use of myocutaneous flaps, were of low quality.

Conclusion: Biologic mesh-assisted perineal reconstruction is a promising technique to improve wound healing and has comparable complications rates to other techniques. However, there is not enough evidence to support its use in all patients who have undergone ELAPE. Results from high-quality prospective RCTs and national/international collaborative audits are required.

Keywords: ELAPE, extralevator abdominoperineal excision of rectum, extralevator abdominoperineal resection, pelvic floor reconstruction, biological mesh 


\section{INTRODUCTION}

Abdominoperineal excision of the rectum (APER) is used as a treatment modality in patients with rectal cancer where an anterior resection (AR) and an anastomosis cannot be performed (1). Extralevator abdominoperineal excision (ELAPE) involves the en bloc excision of the levator muscles and the rectum, in order to reduce the risk of tumor involvement in the circumferential resection margins (CRMs) and reduce the risk of tumor perforation intraoperatively. This method has been demonstrated as leading to a wider surgical margin and therefore fewer positive CRMs (2-5). Initially, the terminology used was "cylindrical APER" but with refinement and the use of MRI to highlight the area of risk of a positive CRM, the term ELAPE is more appropriate (4). The nomenclature surrounding the technique has been the source of much debate and confusion, with some authors noting that ELAPE is no different from the original description in English by Miles (6). Furthermore, what exactly constitutes "standard" surgery that allows differentiation of ELAPE has come under scrutiny (7).

Volumetric analysis has confirmed that ELAPE does remove more tissue (3), and the wider excision can, however, increase morbidity and wound complications and will require some form of perineal reconstruction (4). Perineal wound problems are reported in up to $57 \%$ of patients undergoing APER (8), although the precise rates following ELAPE are not yet known. Given that ELAPE produces a larger defect in the pelvic floor, leaving only the ischiorectal fat and skin to close the perineal wound; it is presumed that the perineal complication rate is higher. Furthermore, the changes in the proportion of patients having neoadjuvant (chemo)radiotherapy over the time course of ELAPE implementation are incompletely reported in individual studies and in national registries. If the wound fails to heal via primary intention, secondary wound healing can result in prolonged hospital stay that requires intensive wound care.

Various alternative techniques have been described to reconstruct the pelvic floor following ELAPE with the aim to reduce perineal wound complications and hernias. The optimal method of perineal reconstruction remains a matter of debate. Myocutaneous flaps, such as those derived from gluteus maximus $(2,4,9)$, rectus abdominis, and latissimus dorsi muscles $(4,10)$, have been used but are associated with donor-site morbidity, flap necrosis, prolonged operative time, additional resources, and increased cost. Biologic mesh has recently been introduced as an alternative form of reconstruction in order to improve perineal wound healing and reduce perineal hernia rates (11). The mesh is usually placed as an inlay or bridge across the defect in the pelvic floor in close relation to the bony structures and sutured in 1-cm intervals to the origin of the levator muscles laterally (12). [Figure 1 (13)] The mechanism by which the use of a bridging prosthesis reduces perineal wound problems is not clear. It has been suggested that biological mesh allows native cellular ingrowth and promotes tissue remodeling, which in turn reduces perineal wound problems $(14,15)$. Alternatively, the biologic mesh may act as a physical barrier, supporting the pelvic contents (omentum, small bowel, and uterus) and minimizing the pressure on the skin and ischiorectal fat as they heal.

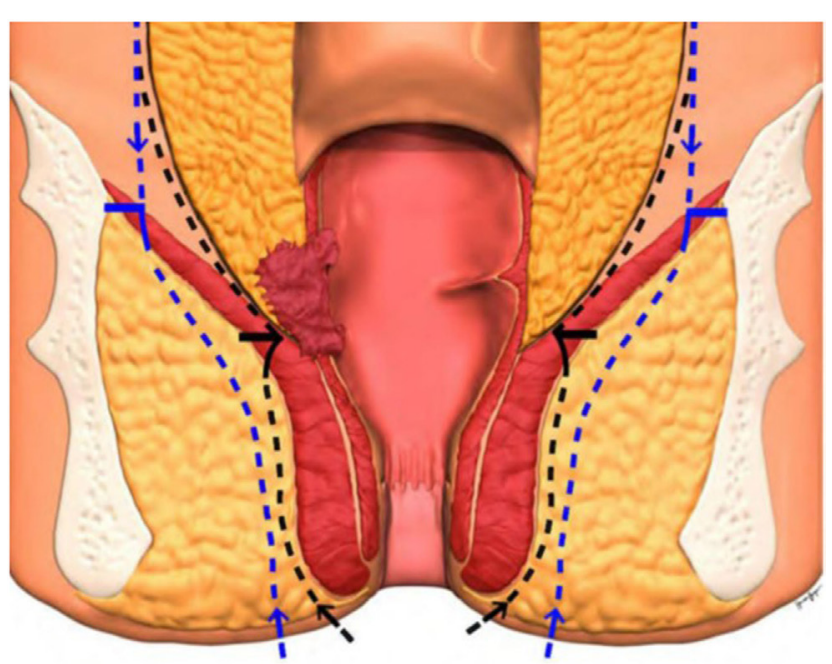

FIGURE 1 | ELAPE technique (13). Black line indicates dissection line of standard APE and blue line ELAPE. Horizontal line indicates meeting point of abdominal and perineal dissection.

Alternative methods for removing the pressure of small bowel that prolapses into the pelvis, directly on the perineum include the following:

(1) Omental pedicle flaps (16-18),

(2) Mobilization of the cecum (9),

(3) Retroversion of the uterus in female (19).

All of the above techniques are designed to close off the dead space in the pelvis, resulting from the removal of the rectum and to keep the small bowel out of the pelvis. Of these methods, the most widely established is the omental pedicle. However, these techniques largely related to an era of open surgery, and they have mostly been abandoned with the move to laparoscopic and other minimally invasive techniques and are not representative of contemporary practice. Omental pedicles are associated with perineal wound complication rates of $14-18 \%$ and decreased wound dehiscence in comparison to primary closure $(16,18)$ whereas others show no advantage to this technique (20). Mobilization of the cecum is uncommon and evidence is limited to case reports (9). Retroversion of the uterus involves retroverting the uterus and securing it to the bony pelvis at a level where it obliterates the pelvis, with the use of non-absorbable suture material (19). This can be achieved via the abdominal or perineal wound, although it has been associated with dyspareunia and positional menstruation (19).

The aim of this review is to provide an overview of the evidence for the use of biologic mesh in the reconstruction of the pelvic floor after extralevator abdominoperineal excision.

\section{METHODS}

A systematic search of PubMed was conducted using the search terms: "ELAPE" "extralevator abdominoperineal excision of rectum," or "extralevator abdominoperineal resection" in order to 
identify studies evaluating the use of biologic mesh for reconstruction of the pelvic floor. Titles, abstracts, and full texts were analyzed for studies reporting on the use of biologic mesh for reconstruction of the pelvic floor. Inclusion criteria were studies that used biologic mesh for perineal reconstruction. Studies were excluded if only synthetic mesh was used or if there was no mention of a mesh. Furthermore, studies on patients under the age of 18 were excluded as well as non-English language studies, technical tips, conference abstracts, or duplicates series from the same research group. Overall, the search yielded 17 studies for analysis after the exclusion of review articles. The study characteristics are presented (Table 1).

\section{RESULTS}

There were 15 case series, one randomized control trial (RCT), and one case report identified. A biologic mesh was used in perineal reconstruction in 463 cases. The different types of biologic mesh used were cross-linked porcine dermal collagen (Permacol ${ }^{\mathrm{TM}}$ ) in 206 cases, 44 using porcine intestinal submucosa (Surgisis@), 136 using human acellular dermal matrix, and 9 using a combination of PermacolTM and Surgisis $\odot$. Two studies did not specify the type of biologic mesh used.

\section{Perineal Hernia}

There were 41 perineal hernias reported, but rates were not consistently reported in all studies. In those studies that did report perineal hernia rates, it was difficult to delineate whether hernias occurred in patients that had perineal reconstruction using a biological or synthetic mesh or a myocutaneous flap.

\section{Perineal Wound Infection/Healing Problems}

Perineal wound infection was reported explicitly in 93 cases, whereas the overall rate of perineal problems was much higher. Perineal sinus and fistulae were reported in 26 cases, with a further 11 cases of perineal hematoma or seroma. Some studies have described "perineal wound complications" but not specified whether they were related to infection, dehiscence, hernia, or pain (Table 1).

The most common complications were perineal wound infection and perineal sinus. However, there are no standardized measures for reporting perineal outcomes of any type following ELAPE. Definitions of wound infection, wound healing problems, perineal herniation, pain measurement, and functional status assessment are inconsistent between studies, thus limiting comparisons.

There are very few studies comparing the use of biologic mesh for perineal reconstruction for ELAPE. Two case series compared biologic mesh with myocutaneous flaps and one series compared laparoscopic ELAPE with laparoscopic and open APER. However, they are all of low-level evidence (level 4). Only one RCT was identified that compared patients undergoing ELAPE with perineal reconstruction using a biological mesh, with patients undergoing a conventional APER with no mesh. There was no significant difference in perineal hernia rates or perineal wound infections between the two groups.

\section{DISCUSSION/SUMMARY}

The use of ELAPE over conventional APER is becoming more widespread despite the reservations of some (13), and the optimal method of perineal wound closure remains a topic of discussion. The reported results of primary closure of the perineal defect are poor (34) and most surgeons performing ELAPE opt for an adjunct (35). The literature analyzed suggests that perineal closure using a biologic mesh produces wound infection and complication rates that are comparable to other methods of reconstruction, such as myocutaneous flaps. Myocutaneous flap reconstruction using a vertical rectus abdominis (VRAM), gracilis, or the gluteus maximus, however, has short-term disadvantages, such as longer operative times and the need for plastic surgical expertise, resulting in higher operative costs, flap necrosis, wound complications at the donor site, and longer bed rest (15). Longer term incisional hernias at the VRAM donor site and reduced abdominal wall strength have been reported (36). Biologic mesh reconstruction avoids all of these potential complications.

Synthetic non-absorbable mesh is associated with high infection rate in contaminated fields and consequently is considered by many to be contra-indicated for use in perineal reconstruction following ELAPE (37). The role of newer, absorbable synthetic meshes is, as yet, unclear. Biologic meshes are composed of an acellular collagen matrix that is believed to allow tissue regeneration, neovascularization, repopulation with fibroblasts, and therefore provides a scaffold for tissue incorporation $(15,23)$. This is thought to reduce the rate of infection. However, the overall volume and quality of evidence available regarding biologic mesh use for perineal reconstruction following ELAPE is poor, with observational retrospective studies predominating. There have been some attempts at comparative studies, but these too have been of low quality with a high risk of bias and confounding factors. Head-to-head randomized trials or high-quality prospective cohort studies comparing biological with synthetic mesh, types of biologic mesh, and biologic mesh with (myo)fasciocutaneous flaps are also lacking, partly because there is no consensus among surgeons as to the optimal biologic mesh or optimal tissue flap. Trials directly comparing any technical adjunct to primary closure alone as a control arm may be difficult to perform in light of the lack of equipoise among surgeons and possibly even unethical given the reported poor results of primary closure. Furthermore, there does not appear to be a consensus in the studies regarding perineal outcome reporting. There are a variety of different end points recorded across the studies, such as perineal defect size, blood loss, and operating time. There needs to be a focus on standardized definitions and reporting of perineal healing rates, perineal hernia, and functional outcomes following ELAPE (38).

Jensen et al. also examined the long-term follow-up for patients undergoing pelvic floor reconstruction with a biologic mesh following ELAPE (25). As well as low perineal hernia rates, there was no major restriction in movement or sitting. Chronic pain had resolved in all patients at a median of 8 months, and there was no major limitation to walking. However, other studies evaluating quality-of-life scores using validated tools (11) demonstrated a favorable comparison to the reference population of patients with colorectal cancer who had undergone a standard 
TABLE 1 | Reconstruction of the pelvic floor after ELAPE.

\begin{tabular}{|c|c|c|c|c|c|c|c|c|c|c|}
\hline Reference & $\begin{array}{l}\text { Study } \\
\text { design }\end{array}$ & No. of pts & Age & $\operatorname{Sex}(M: F)$ & Patient characteristics & Material used & Intervention & $\begin{array}{l}\text { Follow-up } \\
\text { (months) }\end{array}$ & Complications & LoE \\
\hline $\begin{array}{l}\text { Christensen } \\
\text { et al. (21) }\end{array}$ & Case series & 57 & $\begin{array}{l}\text { FLAP: } 67.8 \\
(32.7-86.2) \\
\text { MESH: } 69.7 \\
(48.7-84.5)\end{array}$ & $\begin{array}{l}11: 22 \\
10: 14\end{array}$ & $\begin{array}{l}52 \text { primary rectal cancer } \\
5 \text { local recurrence } \\
48 \text { patients (84\%) } \\
\text { received neoadjuvant } \\
\text { CRT }\end{array}$ & $\begin{array}{l}\text { Gluteal flaps: } 33 \\
\text { Permacol: } 24\end{array}$ & $\begin{array}{l}\text { ELAPE for low rectal } \\
\text { cancer }\end{array}$ & $\begin{array}{l}\text { Median follow- } \\
\text { up: gluteal flap: } \\
3.2 \text { years }(1.7-4.3) \\
\text { Biologic mesh: } 1.7 \\
(0.4-2.2) \text { years }\end{array}$ & $\begin{array}{l}\text { Gluteal flap vs. biologic } \\
\text { Perineal hernia: } 7 \text { vs. } 0, P<0.01 \\
\text { Infectious complications: } 2(17 \%) \\
\text { vs. } 4(6 \%), P<0.26 \\
1 \text { patient per group with a } \\
\text { persistent perineal sinus }\end{array}$ & 4 \\
\hline $\begin{array}{l}\text { Dalton et al. } \\
\text { (22) }\end{array}$ & Case series & 31 & $\begin{array}{l}\text { Mean } \\
66.8 \pm \mathrm{SD} \\
11.4 \text { years }\end{array}$ & $8: 23$ & Neoadjuvant CRT: 14 & $\begin{array}{l}\text { VRAM flap: } 1 \\
\text { Permacol: } 30\end{array}$ & Open ELAPE & Median: 20 (0-45) & $\begin{array}{l}\text { Breakdown of perineal wound: } 6 \\
\text { Skin paddle necrosis of a VRAM } \\
\text { flap: } 1 \\
\text { Perineal wound hematoma: } 1 \\
\text { Minor wound discharge: } 9\end{array}$ & 4 \\
\hline $\begin{array}{l}\text { Han et al. } \\
\text { (23) }\end{array}$ & Case series & 12 & $68(49-80)$ & $7: 5$ & $\begin{array}{l}\text { Ultra low rectal cancer. } \\
\text { Neoadjuvant CRT: } 3\end{array}$ & HADM & Cylindrical APR-open & Median: 8 (2-16) & $\begin{array}{l}\text { Asymptomatic seroma: } 1 \\
\text { Perineal wound infection: } 1\end{array}$ & 4 \\
\hline \multirow[t]{2}{*}{$\begin{array}{l}\text { Han et al. } \\
(14)\end{array}$} & $\begin{array}{l}\text { Open label } \\
\text { RCT }\end{array}$ & 67 & $\begin{array}{l}63 \text { median } \\
(44-81)\end{array}$ & $20: 15$ & Neoadjuvant therapy: 10 & HADM & ELAPE: 35 & Median: 29 (12-48) & $\begin{array}{l}\text { Bowel perforation: } 2 \\
\text { Perineal wound infection: } 4 \\
\text { Perineal seroma: } 4 \\
\text { Peristomal hernia: } 16 \\
\text { Abdominal wound infection: } 2 \\
\text { Perineal herniation: } 5\end{array}$ & 2 \\
\hline & & & 68 (32-84) & $21: 11$ & Neoadjuvant therapy: 9 & None & APER: 32 & Median: 22 (14-46) & $\begin{array}{l}\text { Bowel perforation: } 5 \\
\text { Perineal wound infection: } 6 \\
\text { Peristomal hernia: } 13 \\
\text { Abdominal wound infection: } 3 \\
\text { Perineal herniation: } 4\end{array}$ & \\
\hline $\begin{array}{l}\text { Han et al. } \\
\text { (24) }\end{array}$ & $\begin{array}{l}\text { Multicenter } \\
\text { prospective } \\
\text { cohort study } \\
\text { (case series) }\end{array}$ & 109 (102) & $\begin{array}{l}61 \text { years } \\
(27-78)\end{array}$ & $60: 42$ & & HADM & $\begin{array}{l}\text { Biological mesh: } 83 \\
(81.4 \%) \\
\text { Primary closure: } 19 \\
(18.6 \%)\end{array}$ & 44 median (18-68) & $\begin{array}{l}\text { Biological mesh } \\
\text { Perineal wound complications: } 15 \\
\text { Infection: } 5 \\
\text { Seroma: } 5 \\
\text { Hernia: } 4 \\
\text { Abdominal wound infection: } 3 \\
\text { Primary closure } \\
\text { Perineal wound complications: } 9 \\
\text { Infection: } 3 \\
\text { Seroma: } 1 \\
\text { Hernia: } 2 \\
\text { Wound dehiscence: } 3 \\
\text { Chronic sinus: } 1 \\
\text { Abdominal wound infection: } 2\end{array}$ & 4 \\
\hline
\end{tabular}


TABLE 1 | Continued

\begin{tabular}{|c|c|c|c|c|c|c|c|c|c|c|}
\hline Reference & $\begin{array}{l}\text { Study } \\
\text { design }\end{array}$ & No. of pts & Age & Sex (M:F) & Patient characteristics & Material used & Intervention & $\begin{array}{l}\text { Follow-up } \\
\text { (months) }\end{array}$ & Complications & LoE \\
\hline $\begin{array}{l}\text { Jensen } \\
\text { et al. (25) }\end{array}$ & Case series & $\begin{array}{c}53-31 \\
\text { agreed to } \\
\text { long-term } f / u\end{array}$ & $\begin{array}{l}69(33-83) \\
\text { median }\end{array}$ & $33: 20$ & Neoadjuvant CRT: 23 & Permacol & $\begin{array}{l}6 \text { planned open } \\
47 \text { laparoscopic of which } \\
7 \text { converted to open }\end{array}$ & Median: 36 (1-67) & $\begin{array}{l}\text { Perineal hernia: } 3 \\
\text { Fistuale: } 11 \\
\text { Perineal abscess: } 4 \\
\text { Superficial wound infections: } 4 \\
\text { Removal of mesh: } 1 \\
\text { Implantation of new mesh: } 1\end{array}$ & 4 \\
\hline $\begin{array}{l}\text { Kipling et al. } \\
\text { (26) }\end{array}$ & Case series & 28 & $\begin{array}{l}70 \\
(52-81 \text { years }) \\
\text { median }\end{array}$ & $20: 8$ & $\begin{array}{l}\text { Neoadjuvant therapy } \\
\text { None: } 9 \text { (32\%) } \\
\text { Short course: } 2(7 \%) \\
\text { Long course: } 17(61 \%)\end{array}$ & Permacol & Lap ELAPE, 5 conversions & Median 38 (23-66) & $\begin{array}{l}\text { Bowel perforation: } 1 \\
\text { Persistent perineal sinus at } \\
6 \text { months: } 1 \\
\text { Delayed healing of the perineal } \\
\text { wound: } 1\end{array}$ & 4 \\
\hline \multirow[t]{2}{*}{$\begin{array}{l}\text { Peacock } \\
\text { et al. (15) }\end{array}$} & $\begin{array}{l}\text { Case series } \\
\text { (comparative) }\end{array}$ & 15 & $\begin{array}{l}68 \text { median } \\
(48-74)\end{array}$ & $4: 1$ & $\begin{array}{l}\text { Long-course CT/RT: } 4 \\
\text { Long-course RT: } 1\end{array}$ & VRAM: 5 & Cylindrical APER & Median: 29 (23-35) & $\begin{array}{l}\text { Perineal wound infection (wound } \\
\text { dehiscence): } 1 \\
\text { Flap necrosis: } 1 \\
\text { Wound hematoma: } 1\end{array}$ & 4 \\
\hline & & & $\begin{array}{l}57 \text { median } \\
(47-68)\end{array}$ & $9: 1$ & $\begin{array}{l}\text { Long-course CT/RT: } 6 \\
\text { Long-course RT: } 2 \text { (not } \\
\text { suitable for CT): } 2\end{array}$ & Surgisis: 10 & & $13(3-27)$ & $\begin{array}{l}\text { Perineal sinus: } 1 \\
\text { Superficial perineal wound } \\
\text { infection: } 2 \\
\text { Abscess/collection: } 3\end{array}$ & \\
\hline $\begin{array}{l}\text { Peacock } \\
\text { et al. (27) }\end{array}$ & Case series & 34 & $\begin{array}{l}\text { Median } \\
62 \text { years } \\
(40-77)\end{array}$ & $27: 7$ & $\begin{array}{l}\text { Long-course CRT: } 26 \\
\text { Long-course RT (not } \\
\text { suitable for CT): } 2 \\
\text { Not required/declined: } 6\end{array}$ & Surgisis: & Cylindrical APER & Median: $21(1-54)$ & $\begin{array}{l}\text { Perineal sinus: } 5 \\
\text { Superficial perineal wound } \\
\text { infection: } 3 \\
\text { Abscess/collection: } 3 \\
\text { Parastomal hernia: } 1\end{array}$ & 4 \\
\hline $\begin{array}{l}\text { Vaughan- } \\
\text { Shaw et al. } \\
\text { (28) }\end{array}$ & $\begin{array}{l}\text { Case series } \\
\text { (case-control) }\end{array}$ & $\begin{array}{l}16 \\
10\end{array}$ & $\begin{array}{l}71(49-88) \\
72(52-87) \\
72.5(46-89)\end{array}$ & $\begin{array}{l}7: 9 \\
5: 5 \\
8: 2\end{array}$ & $\begin{array}{l}\text { Short-course RT: } 7 \\
\text { Long-course CRT: } 9 \\
\text { Short-course RT: } 7 \\
\text { Long-course CRT: } 2 \\
\text { Short-course RT: } 2 \\
\text { Long-course CRT: } 5\end{array}$ & $\begin{array}{l}9 \text { Permacol/ } \\
\text { Surgisis } \\
\text { (omentoplasty: 7) }\end{array}$ & $\begin{array}{l}\text { Laparoscopic ELAPE: } 14 \\
\text { (1 conversion) } \\
\text { Open: } 2 \\
\text { Lap APER: } 10 \\
\text { Open APER: } 10\end{array}$ & & $\begin{array}{l}\text { Return to theater ( }<30 \text { days): } 2 \\
\text { Perineal wound complications: } 2 \\
\text { Perineal wound complications: } 5 \\
\text { Perineal hernia: } 2 \\
\text { Infection: } 1 \\
\text { Return to theater ( }<30 \text { days): } 1 \\
\text { In-hospital mortality: } 1 \\
\text { Perineal wound complications: } 2\end{array}$ & 4 \\
\hline $\begin{array}{l}\text { Wille- } \\
\text { Jørgensen } \\
\text { et al. (29) }\end{array}$ & Case series & 11 & $\begin{array}{l}63 \text { median } \\
(51-77)\end{array}$ & $7: 4$ & Neoadjuvant CRT: 6 & Permacol & $\begin{array}{l}\text { Laparoscopic APER: } 9 \\
\text { (2 conversions) Open } \\
\text { APER: } 2\end{array}$ & Median: $12(3-18)$ & $\begin{array}{l}\text { Mesh removal 2nd to infection: } 1 \\
\text { Rectal perforation: } 1 \\
\text { Long-lasting perineal pain: } 6 \\
\text { Fistula: } 1\end{array}$ & 4 \\
\hline $\begin{array}{l}\text { Chi et al. } \\
\text { (30) }\end{array}$ & Case series & 6 & Mean: 69 & $4: 2$ & Neoadjuvant CRT 4 & HADM & & Mean: 5 (2-19) & Surgical site infection: 2 & 4 \\
\hline
\end{tabular}


TABLE 1 | Continued

\begin{tabular}{|c|c|c|c|c|c|c|c|c|c|c|}
\hline Reference & $\begin{array}{l}\text { Study } \\
\text { design }\end{array}$ & No. of pts & Age & Sex (M:F) & Patient characteristics & Material used & Intervention & $\begin{array}{l}\text { Follow-up } \\
\text { (months) }\end{array}$ & Complications & LoE \\
\hline $\begin{array}{l}\text { Palmer } \\
\text { et al. (31) }\end{array}$ & Case series & 193 & $\begin{array}{l}66 \text { median } \\
(28-87)\end{array}$ & $81: 112$ & $\begin{array}{l}\text { Neoadjuvant CRT: } 91 \\
\text { RT alone: } 92 \\
\text { Locally advanced tumor } \\
\text { on MRI (T4)-126 (65\%) }\end{array}$ & $\begin{array}{l}\text { Perineal closure } \\
\text { Gluteal flap: } 99 \\
\text { (51) } \\
\text { Biological mesh: } \\
66 \text { (34) } \\
\text { Closure directly: } \\
28(15)\end{array}$ & $\begin{array}{l}\text { Pelvic exenteration: 25, } \\
\text { extended resection with } \\
\text { parts of other organs: } 56 \\
\text { ELAPE alone: } 112\end{array}$ & Median $31(0-156)$ & $\begin{array}{l}\text { Intra-operative perforation: } 19 \\
\text { 30-day postoperative mortality: } 6\end{array}$ & 4 \\
\hline \multirow[t]{2}{*}{$\begin{array}{l}\text { West et al. } \\
\text { (4) }\end{array}$} & $\begin{array}{l}\text { Retrospective } \\
\text { case series } \\
\text { (multicenter) }\end{array}$ & 176 & $\begin{array}{l}66(58-73) \\
\text { Median }\end{array}$ & $\begin{array}{l}\text { 116:54 } \\
\text { 6-unknown }\end{array}$ & $\begin{array}{l}\text { Neoadjuvant RT } \\
\text { Yes: } 135 \\
\text { No: } 35 \\
\text { Unknown: } 11 \\
\text { Neoadjuvant CT } \\
\text { Given: } 84 \\
\text { Not given: } 81 \\
\text { Unknown: } 11\end{array}$ & $\begin{array}{l}\text { Gluteus maximus: } \\
60 \\
\text { Rectus abdominis: } \\
12 \\
\text { Latissimus dorsi: } 1 \\
\text { Permacol: } 11\end{array}$ & $\begin{array}{l}\text { ELAPE: } 176 \\
\text { Open surgery: } 122 \\
\text { Laparoscopic surgery: } 19 \\
\text { Unknown: } 35\end{array}$ & NS & $\begin{array}{l}\text { Wound complications } \\
\text { Yes: } 57 \\
\text { Infection/breakdown/sinus: } 41 \\
\text { Perineal hernia: } 5 \\
\text { Other: } 11\end{array}$ & 4 \\
\hline & & 124 & $\begin{array}{l}68(57-75) \\
\text { median }\end{array}$ & $87: 37$ & $\begin{array}{l}\text { Neoadjuvant RT } \\
\text { Yes: } 90 \\
\text { No: } 24 \\
\text { Unknown: } 10 \\
\text { Neoadjuvant CT } \\
\text { Given: } 48 \\
\text { Not given: } 66 \\
\text { Unknown: } 10\end{array}$ & & $\begin{array}{l}\text { APER: } 124 \\
\text { Open surgery: } 56 \\
\text { Laparoscopic surgery: } 4 \\
\text { Unknown: } 64\end{array}$ & NS & $\begin{array}{l}\text { Wound complications } \\
\text { Yes: } 11 \\
\text { Infection/breakdown/sinus: } 7 \\
\text { Perineal hernia: } 1 \\
\text { Other: } 3 \\
\text { Unknown: } 26\end{array}$ & \\
\hline $\begin{array}{l}\text { Harries } \\
\text { et al. (32) }\end{array}$ & $\begin{array}{l}\text { Prospective } \\
\text { case series }\end{array}$ & 48 & $\begin{array}{l}\text { Median: } 63 \\
(40-86)\end{array}$ & $36: 12$ & $\begin{array}{l}\text { Neoadjuvant } \\
\text { treatment: } 43\end{array}$ & Permacol & $\begin{array}{l}\text { ELAPE } \\
\text { Lap: } 28 \\
\text { Conversion: } 7 \\
\text { Open: } 23\end{array}$ & Median: 27 (1-85) & $\begin{array}{l}\text { Specimen perforation: } 3 \text { (6.4\%) } \\
\text { Unhealed at } 6 \text { months: } 4 \text { (8.3\%) } \\
\text { Perineal sinus: } 7 \\
\text { Abdominal wound dehiscence: } 1 \\
\text { Ureteric injury: } 1 \\
\text { Radiological drainage of pelvic } \\
\text { collections: } 2 \\
\text { Perineal wound infections: } 9\end{array}$ & 4 \\
\hline $\begin{array}{l}\text { Kavanagh } \\
\text { et al. (33) }\end{array}$ & Case report & 1 & 72 & $0: 1$ & Long-course CRT & Permacol & Lap ELAPE & 12 & NS & 4 \\
\hline $\begin{array}{l}\text { Sayers et al. } \\
\text { (34) }\end{array}$ & Case series & 54 & $\begin{array}{l}\text { Median: } 69.5 \\
(31-90)\end{array}$ & 40:14 & Neoadjuvant CRT: 52 & $\begin{array}{l}\text { Primary closure: } \\
46 \\
\text { Bio: } 2 \\
\text { FLAP: } 6 \text { (VRAM: } 5 \\
\text { Gracilis: } 1 \text { ) }\end{array}$ & $\begin{array}{l}\text { Lap ELAPE: } 20 \\
\text { Open: } 34\end{array}$ & Median: 38 (9-61) & $\begin{array}{l}\text { Perineal complications: } 24 \\
\text { Perineal hernia: } 14 \\
\text { Perineal hematoma: } 1 \\
\text { Infected myocutaneous flap: } 1 \\
\text { Total dehiscence of the } \\
\text { perineum: } 1\end{array}$ & 4 \\
\hline
\end{tabular}

APER, abdominoperineal excision of the rectum; CRT, chemoradiotherapy; CT, chemotherapy; ELAPE, extralevator abdominoperineal excision; HADM, human acellular dermal matrix; LoE, level of evidence; RCT, randomized controlled trial; RT, radiotherapy; VRAM, vertical rectus abdominis muscle. 
APE, whereas patients who had undergone flap reconstruction had a lower quality of life score (11).

Of note, a number of studies from Beijing have been included for analysis. The three studies include patients managed over an approximately 3-year period, and there is overlap of the studies within the time period, therefore suggesting some replication. One study is classified as a case series (23), the second a RCT (14), and the third another case series (24). It is unclear as to whether these three studies are from the same patient group or three different cohorts.

\section{CONCLUSION}

Overall, the use of a biologic mesh to close perineal defects has comparable complications rates to myocutaenous flaps but

\section{REFERENCES}

1. Perry WB, Connaughton JC. Abdominoperineal resection: how is it done and what are the results? Clin Colon Rectal Surg (2007) 20(3):213-20. doi:10.1055/s-2007-984865

2. Holm T, Ljung A, Häggmark T, Jurell G, Lagergren J. Extended abdominoperineal resection with gluteus maximus flap reconstruction of the pelvic floor for rectal cancer. Br J Surg (2007) 94(2):232-8. doi:10.1002/bjs.5489

3. West NP, Finan PJ, Anderin C, Lindholm J, Holm T, Quirke P. Evidence of the oncologic superiority of cylindrical abdominoperineal excision for low rectal cancer. J Clin Oncol (2008) 26(21):3517-22. doi:10.1200/ JCO.2007.14.5961

4. West NP, Anderin C, Smith KJ, Holm T, Quirke P. European Extralevator Abdominoperineal Excision Study Group. Multicentre experience with extralevator abdominoperineal excision for low rectal cancer. Br J Surg (2010) 97(4):588-99. doi:10.1002/bjs.6916

5. Shihab OC, Heald RJ, Holm T, How PD, Brown G, Quirke P, et al. A pictorial description of extralevator abdominoperineal excision for low rectal cancer. Colorectal Dis (2012) 14(10):e655-60. doi:10.1111/j.1463-1318.2012.03181.x

6. Miles WE. A method of performing abdomino-perineal excision for carcinoma of the rectum and of the terminal portion of the pelvic colon (1908). CA Cancer J Clin (1971) 21(6):361-4. doi:10.3322/canjclin.21.6.361

7. Nicholls J. No more 'standard' abdominoperineal excision. Colorectal Dis (2013) 15(11):1329-30. doi:10.1111/codi.12458

8. de Bruin AF, Gosselink MP, Wijffels NA, Coene PP, van der Harst E. Local gentamicin reduces perineal wound infection after radiotherapy and abdominoperineal resection. Tech Coloproctol (2008) 12(4):303-7. doi:10.1007/ s10151-008-0440-8

9. Habib K. Prevention of recurrent small bowel obstruction resulting from pelvic adhesions in patients who have previously undergone abdominoperineal excision of the rectum. Tech Coloproctol (2014) 18(12):1179-80. doi:10.1007/ s10151-014-1227-8

10. McMenamin DM, Clements D, Edwards TJ, Fitton AR, Douie WJ. Rectus abdominis myocutaneous flaps for perineal reconstruction: modifications to the technique based on a large single-centre experience. Ann R Coll Surg Engl (2011) 93(5):375-81. doi:10.1308/003588411X572268

11. Foster JD, Pathak S, Smart NJ, Branagan G, Longman RJ, Thomas MG, et al. Reconstruction of the perineum following extralevator abdominoperineal excision for carcinoma of the lower rectum: a systematic review. Colorectal Dis (2012) 14(9):1052-9. doi:10.1111/j.1463-1318.2012.03169.x

12. Svane M, Bulut O. Perineal hernia after laparoscopic abdominoperineal resection-reconstruction of the pelvicfloor with abiologicalmesh (Permacol ${ }^{\mathrm{TM}}$ ). Int J Colorectal Dis (2012) 27(4):543-4. doi:10.1007/s00384-011-1253-1

13. Prytz M, Angenete E, Ekelund J, Haglind E. Extralevator abdominoperineal excision (ELAPE) for rectal cancer - short-term results from the Swedish colorectal cancer registry. Selective use of ELAPE warranted. Int J Colorectal Dis (2014) 29(8):981-7. doi:10.1007/s00384-014-1932-9

14. Han JG, Wang ZJ, Wei GH, Gao ZG, Yang Y, Zhao BC. Randomized clinical trial of conventional versus cylindrical abdominoperineal resection for locally may offer advantages, such as shorter operating time and early mobilization, which results in a more cost-effective repair (15). Biologic mesh-assisted perineal reconstruction is a promising technique to improve wound healing, but there is not enough evidence to support its use in all patients who have undergone ELAPE. The results from high-quality prospective RCTs or national/international collaborative audits using statistical process control as a methodology of assessment of improvement are required.

\section{AUTHOR CONTRIBUTIONS}

All authors listed have made substantial, direct, and intellectual contribution to the work and approved it for publication.

advanced lower rectal cancer. Am J Surg (2012) 204(3):274-82. doi:10.1016/j. amjsurg.2012.05.001

15. Peacock O, Pandya H, Sharp T, Hurst NG, Speake WJ, Tierney GM, et al. Biological mesh reconstruction of perineal wounds following enhanced abdominoperineal excision of rectum (APER). Int J Colorectal Dis (2012) 27(4):475-82. doi:10.1007/s00384-011-1325-2

16. Hay JM, Fingerhut A, Paquet JC, Flamant Y. Management of the pelvic space with or without omentoplasty after abdominoperineal resection for carcinoma of the rectum: a prospective multicenter study. The French Association for Surgical Research. Eur J Surg (1997) 163(3):199-206.

17. Moreaux J, Horiot A, Barrat F, Mabille J. Obliteration of the pelvic space with pedicled omentum after excision of the rectum for cancer. Am J Surg (1984) 148(5):640-4. doi:10.1016/0002-9610(84)90342-8

18. Killeen S, Devaney A, Mannion M, Martin ST, Winter DC. Omental pedicle flaps following proctectomy: a systematic review. Colorectal Dis (2013) 15(11):e634-45. doi:10.1111/codi.12394

19. Habib K. Uterus and extralevator abdominoperineal excision: a technique. Dis Colon Rectum (2013) 56(9):1102-4. doi:10.1097/DCR.0b013e31829a2edc

20. O'Leary DP. Use of the greater omentum in colorectal surgery. Dis Colon Rectum (1999) 42(4):533-9. doi:10.1007/BF02234183

21. Christensen HK, Nerstrøm P, Tei T, Laurberg S. Perineal repair after extralevator abdominoperineal excision for low rectal cancer. Dis Colon Rectum (2011) 54(6):711-7. doi:10.1007/DCR.0b013e3182163c89

22. Dalton RS, Smart NJ, Edwards TJ, Chandler I, Daniels IR. Short-term outcomes of the prone perineal approach for extra-levator abdomino-perineal excision (elAPE). Surgeon (2012) 10(6):342-6. doi:10.1016/j.surge.2011.10.001

23. Han JG, Wang ZJ, Gao ZG, Xu HM, Yang ZH, Jin ML. Pelvic floor reconstruction using human acellular dermal matrix after cylindrical abdominoperineal resection. Dis Colon Rectum (2010) 53(2):219-23. doi:10.1007/ DCR.0b013e3181b715b5

24. Han JG, Wang ZJ, Qian Q, Dai Y, Zhang ZQ, Yang JS, et al. A prospective multicenter clinical study of extralevator abdominoperineal resection for locally advanced low rectal cancer. Dis Colon Rectum (2014) 57(12):1333-40. doi:10.1097/DCR.0000000000000235

25. Jensen KK, Rashid L, Pilsgaard B, Møller P, Wille-Jørgensen P. Pelvic floor reconstruction with a biological mesh after extralevator abdominoperineal excision leads to few perineal hernias and acceptable wound complication rates with minor movement limitations: single-centre experience including clinical examination and interview. Colorectal Dis (2014) 16(3):192-7. doi:10.1111/codi.12492

26. Kipling SL, Young K, Foster JD, Smart NJ, Hunter AE, Cooper E, et al. Laparoscopic extralevator abdominoperineal excision of the rectum: short-term outcomes of a prospective case series. Tech Coloproctol (2014) 18(5):445-51. doi:10.1007/s10151-013-1071-2

27. Peacock O, Simpson JA, Tou SI, Hurst NG, Speake WJ, Tierney GM, et al. Outcomes after biological mesh reconstruction of the pelvic floor following extra-levator abdominoperineal excision of rectum (APER). Tech Coloproctol (2014) 18(6):571-7. doi:10.1007/s10151-013-1107-7

28. Vaughan-Shaw PG, Cheung T, Knight JS, Nichols PH, Pilkington SA, Mirnezami AH. A prospective case-control study of extralevator 
abdominoperineal excision (ELAPE) of the rectum versus conventional laparoscopic and open abdominoperineal excision: comparative analysis of short-term outcomes and quality of life. Tech Coloproctol (2012) 16(5):355-62. doi:10.1007/s10151-012-0851-4

29. Wille-Jørgensen P, Pilsgaard B, Møller P. Reconstruction of the pelvic floor with a biological mesh after abdominoperineal excision for rectal cancer. Int J Colorectal Dis (2009) 24(3):323-5. doi:10.1007/s00384-008-0607-9

30. Chi P, Chen ZF, Lin HM, Lu XR, Huang Y. Laparoscopic extralevator abdominoperineal resection for rectal carcinoma with transabdominal levator transection. Ann Surg Oncol (2013) 20(5):1560-6. doi:10.1245/ s10434-012-2675-x

31. Palmer G, Anderin C, Martling A, Holm T. Local control and survival after extralevator abdominoperineal excision for locally advanced or low rectal cancer. Colorectal Dis (2014) 16(7):527-32. doi:10.1111/codi.12610

32. Harries RL, Luhmann A, Harris DA, Shami JA, Appleton BN. Prone extralevator abdominoperineal excision of the rectum with porcine collagen perineal reconstruction (Permacol ${ }^{\mathrm{TM}}$ ): high primary perineal wound healing rates. Int J Colorectal Dis (2014) 29(9):1125-30. doi:10.1007/ s00384-014-1963-2

33. Kavanagh DO, Imran H, Almoudaris A, Ziprin P, Faiz O. Dynamic magnetic resonance imaging demonstrates the integrity of perineal reconstruction following cylindrical abdominoperineal excision with reconstruction of the pelvic floor using porcine collagen. Case Rep Med (2012) 2012:752357. doi:10.1155/2012/752357

34. Sayers AE, Patel RK, Hunter IA. Perineal hernia formation following extralevator abdominoperineal excision. Colorectal Dis (2015) 17(4):351-5. doi:10.1111/codi.12843
35. Dabbas N, Adams K, Chave H, Branagan G. Current practice in abdominoperineal resection: an email survey of the membership of the Association of Coloproctology. Ann R Coll Surg Engl (2012) 94(3):173-6. doi:10.1308/0035 88412X13171221589973

36. Daigeler A, Simidjiiska-Belyaeva M, Drücke D, Goertz O, Hirsch T, Soimaru $\mathrm{C}$, et al. The versatility of the pedicled vertical rectus abdominis myocutaneous flap in oncologic patients. Langenbecks Arch Surg (2011) 396(8):1271-9. doi:10.1007/s00423-011-0823-6

37. Choi JJ, Palaniappa NC, Dallas KB, Rudich TB, Colon MJ, Divino CM. Use of mesh during ventral hernia repair in clean-contaminated and contaminated cases: outcomes of 33,832 cases. Ann Surg (2012) 255(1):176-80. doi:10.1097/ SLA.0b013e31822518e6

38. Haapamäki MM, Pihlgren V, Lundberg O, Sandzén B, Rutegård J. Physical performance and quality of life after extended abdominoperineal excision of rectum and reconstruction of the pelvic floor with gluteus maximus flap. Dis Colon Rectum (2011) 54(1):101-6. doi:10.1007/DCR.0b013e3181fce26e

Conflict of Interest Statement: The authors declare that the research was conducted in the absence of any commercial or financial relationships that could be construed as a potential conflict of interest.

Copyright (C) 2016 Alam, Narang, Köckerling, Daniels and Smart. This is an open-access article distributed under the terms of the Creative Commons Attribution License (CC BY). The use, distribution or reproduction in other forums is permitted, provided the original author $(s)$ or licensor are credited and that the original publication in this journal is cited, in accordance with accepted academic practice. No use, distribution or reproduction is permitted which does not comply with these terms. 